\title{
Postpartum depression and its correlation with spiritual health
}

\author{
Mohammadreza Noormohammadi ${ }^{1 *(D)}$, Lobat Jafarzade ${ }^{2}$, Seyed Kamal Solati ${ }^{3}$, Morteza Sedehi ${ }^{4}$, Mohammad Bagheri $^{5}$, Ali \\ Hasanpour Dehkordi6
}

${ }^{1}$ Associate Professor, Department of Islamic Teachings, Shahrekord University of Medical Sciences, Shahrekord, Iran.

${ }^{2}$ Assistant Professor, Department of Obstetrics and Gynecology, Shahrekord University of Medical Sciences, Shahrekord, Iran. ${ }^{3}$ Associate Professor, Department of Psychology, Faculty of Medicine, Shahrekord University of Medical Sciences, Shahrekord, Iran.

${ }^{4}$ Assistant Professor, Department of Epidemiology and Biostatistics, Shahrekord University of Medical Sciences, Shahrekord, Iran.

${ }^{5} \mathrm{MD}$, Department of Medical Sciences, Shahrekord University of Medical Sciences, Shahrekord, Iran.

${ }^{6}$ Associate Professor, School of Allied Medical Sciences, Shahrekord University of Medical Sciences, Shahrekord, Iran.

*Corresponding Author: Mohammadreza Noormohammadi, Associate Professor, Department of Islamic Teachings, Shahrekord University of Medical Sciences, Shahrekord, Iran, Tel: 09133815984, E-mail: noormohammadi33@yahoo.com

\begin{abstract}
Background and aims: Postpartum depression has a high prevalence in communities and needs great financial resources. On the other hand, the world views the spiritual health as a new and complementary dimension of health. The aim of this study was to investigate the correlation between postpartum depression and spiritual health.

Materials and Methods: This correlational-analytic study was conducted on 152 women who gave birth 8 months ago, referred to Hajar Hospital, Imam Ali hospital, Molavi clinic, or other health centers of Shahrekord, and were also satisfied with participating in the study. They were selected by the simple sampling method and completed the Palutzian and Ellison Spiritual Health Questionnaire. Finally, the collected data were entered in SPSS software and descriptive statistics in addition to the Pearson correlation coefficient and K2 test were used to analyze the data.

Results: In the present study, the mean age of the subjects was 29.15 (SD =5.45) with the range of 16-45 years. The mean scores of spiritual health and depression were obtained 96.3 and 8.94 , respectively. The results of this study showed that the Pearson correlation coefficient was $-75.5 \%(P<0.001)$, demonstrating an inverse correlation between the scores of postpartum depression and spiritual health.

Conclusion: In general, the correlation between postpartum depression and spiritual health was an inverse correlation and the level of depression decreased by an increase in spiritual health. It can be concluded that without the need for specific treatments, the level of postpartum depression can be reduced by increasing the level of spiritual health.

Keywords: Spiritual health, Postpartum depression, Health, Psychiatric disorders
\end{abstract}

Received: 16 June 2019, Accepted: 16 September 2019, ePublished: 28 February 2020

\section{Introduction}

Depression is one of the most important causes of disability in all countries and among the most common psychiatric disorders. It is also considered as a major health problem in all cultures (1). In addition, depression is a mood which can be persistent or unstable and can affect many aspects of an individual's life and interfere with it. Further, depression is characterized by symptoms such as sadness, failure, discomfort, excitement, and stress, along with the lack of interest in everything and even everybody. In fact, the patient couches his or her discomfort in these symptoms (2). Investigations indicated that about 19 million people experience depression every year in the United States (about 1.3\% of the adult population) and about two-thirds of the people do not receive the required help. These patients complain more about physical discomforts, the lack of energy, or feeling weak and are usually left undiagnosed (3). Based on the studies conducted in Iran, about 7 million people suffer from a kind of mental disorder and about $15 \%-25 \%$ of the population of Iran experience mild to severe depression. This is a common disorder and $15 \%$ of people are faced with at least once in their lives. It is also predicted that the number of depressed people would increase owing to the pressures caused by social and environmental changes and an increase in some physical diseases (4). 
The prevalence of mental disorders in females is twice as much as males due to several social reasons including gender discrimination $(5,6)$. Postpartum depression is one of the mental disorders. Further, the postpartum period is so stressful that may cause a new mental disorder, recurrence, or exacerbation of a mental disorder (7). In general, postpartum mental disorders are classified into three groups including sadness, depression, and psychosis (8). More than $85 \%$ of mothers experience postpartum depression and sadness. Its maximum level is experienced in the first week, especially on the second and third day after childbirth. It usually disappears by itself, but if persistent, its severity increases and the postpartum depression will develop in the person (9).

Nowadays, the importance of mental health is emphasized worldwide and various studies have confirmed its role in personal and social life. In this regard, the World Health Organization announced the year 2001 as the global year of mental health. The increasing prevalence of various types of mental disorders in the world requires paying attention to mental illnesses and their prevention (10). This disorder has a significant and negative effect on the quality of the mother's life. Mothers with postpartum depression also have problems in performing their social activities and housekeeping duties, as well as their personal functioning (11). If it is not treated, the person will suffer from its problems even up to menopause. Nowadays, the significant prevalence of postpartum depression in developed and developing countries, along with its adverse effects on mothers and family members, especially on all dimensions of child growth and development, has changed this disorder to a major public health problem around the world. Besides, human beings are faced with rapid changes in most communities regarding all life dimensions which are significantly reflected in their physical and mental health (12).

The necessity of performing this project is because postpartum depression is one of the mental disorders. The postpartum period is so stressful that may cause a new mental disorder, recurrence, or the exacerbation of a mental disorder. Facing with physical and psychological problems may result in acute and severe crises in a man's health and his vulnerability, but it increases his individual and spiritual growth (13). Postpartum mental diseases are important in three aspects. It is predictable at birth and occurs at the time of birth. Further, it causes severe psychological stress on the woman and negatively impacts her specific tasks. Finally, disability in creating interaction with children will cause unpleasant complications for the promotion of children's social and emotional cognitive abilities.

Spirituality as a human dimension is also considered increasingly by recognizing its role in human progress so that it is now regarded as an essential component of clinical care for meeting the mental needs of the patients (14). Moreover, as one of the most important aspects of human existence, it has an essential correlation with people (15). Depression is the most common postpartum psychological disorder. Therefore, the current study aimed to evaluate the correlation between postpartum depression and the level of spiritual health. In addition, the specific goal of the present study was to detect the level of postpartum depression. Eventually, the other specific goal of the present study was to determine the level of postpartum mental health.

\section{Methods}

The present study was a correlational type of an analytical study that was conducted in 2016. Totally, 152 women who gave birth and referred to Hajar hospital $(n=53)$, Imam Ali Clinic ( $n=28)$, Moulavi clinic $(n=21)$, and other health centers of Shahrekord $(n=50)$ during the previous 8 months and showed the consent to participate in the study were selected using the convenience sampling method. Sampling was computed by the following formula.

$$
\begin{aligned}
& N=\frac{4\left(Z_{1-\frac{\alpha}{2}}+Z_{1-\beta}\right)^{2}}{\left(\ln \frac{1+r}{1-r}+\ln \frac{1+P}{1-P}\right)}+3 N=\frac{4\left(Z_{1-\frac{\alpha}{2}}+Z_{1-\beta}\right)^{2}}{\left(\ln \frac{1+\gamma}{1-r}+\ln \frac{1+P}{1-P}\right)}+3=149 \\
& Z_{1-\beta}=1.28 \\
& Z_{1-\frac{\alpha}{2}}=1.96
\end{aligned}
$$

\section{$1-\beta=90 \%, 1-\alpha=95 \%, R=70 \%, P<0.05$}

The inclusion criteria included mothers who referred to the above-mentioned centers during the cited 8-month time interval after the accouchement. On the other hand, the exclusion criteria were incomplete completion of the questionnaire, a history of depression and psychological diseases, and unwanted pregnancy. It should be noted that although some intervening variables were impossible to control, they had negligible effects. Such variables included some other psychological factors like anxiety and stress in addition to some environmental conditions like very cold or hot weather and stormy and cloudy conditions.

In the present study, Palutzian and Ellison's Spiritual Health Scale was used to evaluate the spiritual health. This scale was designed by Palutzian and Ellison in 1982 and used in numerous studies. Its reliability was reported 0.82 using Cronbach's alpha. This scale consisted of 20 questions assessing religious $(n=10)$ and existential $(n=10)$ health. Furthermore, it was scored on a 6-point Likert-type scale ("I strongly disagree $=1$ ", "I disagree" $=2$, "I fairly disagree" $=3$, “I fairly agree" =4, “I agree" $=5$, and "I strongly agree" =6) and questions 1, 2, 5, 6, 9, 12, 14, 16, and 18 were scored inversely. Additionally, a score within the range of 20-40, 41-99, and 100-120 was considered as low, moderate, and high spiritual health, respectively (16). Moreover, Edinburgh Depression Questionnaire was applied to assess the presence or absence of depression in 
mothers. The validity of this questionnaire was confirmed and its Cronbach alpha was reported as 0.82-0.95 in various studies (17). This instrument contained 10 multiple choice questions scored on a Likert-type scale from 0 to 3 . The minimum and maximum scores were 0 and 30. In this study, the cutoff point of 12 was considered as depression. The cutoff score in the Edinburgh Postnatal Depression Scale in an Iranian sample was also considered 12 (18). It should be mentioned that this questionnaire has high validity and reliability and is a suitable tool for measuring postpartum depression. The Cronbach alpha of the translated version of this questionnaire was reported as 0.82-0.90. Before administering the questionnaire, the research goals were explained to the mothers who referred to health centers and gave their consent to participate in the study. The samples were then divided into two groups of depressed (the score equal to or higher than 12 in the Edinburgh test) and non-depressed (the score lower than 12) people based on a questionnaire and a psychiatric diagnosis. Finally, the mothers completed the Paloutzian and Ellison's spiritual Health Questionnaire (1982).

\section{Statistical Analysis}

Descriptive statistics, along with Pearson correlation and chi-square tests were used to analyze the data by SPSS software. The Pearson correlation coefficient and the significance level of $P<0.05$ were used to determine the presence or absence and the direction of the correlation between the samples.

Table 1. Descriptive characteristics of studied women

\begin{tabular}{lccc}
\hline Characteristics & $\begin{array}{c}\text { Spiritual Health } \\
\text { Score }\end{array}$ & $\begin{array}{c}\text { Depression } \\
\text { Score }\end{array}$ & $\begin{array}{c}\text { Number of } \\
\text { Accouchement }\end{array}$ \\
\hline Mean & 96.30 & 8.49 & 1.81 \\
\hline Median & 100 & 8 & 2 \\
\hline SD & 17.81 & 5.40 & 1.01 \\
Variance & 317.24 & 29.19 & 1.02 \\
Minimum & 37 & 0 & 1 \\
\hline Maximum & 120 & 25 & 6 \\
\hline Total & 152 & 152 & 152 \\
\hline Note. SD: Standard deviation. & & \\
\hline
\end{tabular}

\section{Results}

In the present study, the mean age of the subjects was 29.15 $(\mathrm{SD}=5.45)$ within the range of $16-45$ years. Overall, 122 out of 152 studied women had no depression and 3 had a drug use background (2\%). The mean scores of spiritual health and depression were 96.3 and 8.94, respectively (Table 1).

Out of 122 non-depressed women, 1 had a background of chronic diseases. From all women who completed the questionnaires, 41 cases obtained a score of 12 or higher. Out of these 41 women, 7 had a history of abortion, 1 had a chronic disease and depression history simultaneously, and 1 had a history of abortion and depression simultaneously. From 122 non-depressed women, 48 cases had moderate spiritual health and 74 of them had high spiritual health. Out of 30 depressed women, 2 cases had low spiritual health and 28 of them had moderate spiritual health (Table 2).

The results of the Pearson correlation showed a significant and inverse correlation between postpartum depression and spiritual health $(\mathrm{r}=-0.75, P<0.001)$.

\section{Discussion}

The results of the study suggested that a great number of women experienced depression during pregnancy and required specialized and intensive care. Further, the results of this research revealed a significant correlation between reducing postpartum depression and increasing spiritual health. A previous study examined the correlation between spiritual health and life expectancy in cancer patients and demonstrated that providing long-term spiritual care to patients would increase life expectancy and improve the mental status of the patients (19), which is consistent with the findings of our study. In another study, Abbasi et al also showed that spiritual components have positive preventive effects although more studies are required to clarify the correlation between spiritual and physical health. Based on the results, the prevention of diseases cannot be separated from spiritual care (20), which is in agreement with the results of the present study. Furthermore, Ramazani investigated patients with type 2 diabetes and reported that spiritual, existential, and religious health had a significant correlation with the quality of life and dimensions of

Table 2. Spiritual health level of depressed and non-depressed women

\begin{tabular}{|c|c|c|c|c|c|}
\hline \multirow{2}{*}{ Variables } & & \multicolumn{3}{|c|}{ Level of Spiritual Health } & \multirow{2}{*}{ Total } \\
\hline & & High & Moderate & Low & \\
\hline \multirow{2}{*}{ Non-depressed women } & No. & 74 & 48 & 0 & 122 \\
\hline & $\%$ & 60.7 & 39.3 & 0 & 100 \\
\hline \multirow{2}{*}{ Depressed women } & No. & 0 & 28 & 2 & 30 \\
\hline & $\%$ & 0 & 93.3 & 6.7 & 100 \\
\hline \multirow{2}{*}{ Total } & No. & 74 & 76 & 2 & 152 \\
\hline & $\%$ & 48.7 & 50 & 1.3 & 100 \\
\hline
\end{tabular}


physical, mental, social, and environmental health (21). They also emphasized the importance of strengthening the spiritual dimension of health as an important factor in improving the quality of life of these patients. The results of the above-mentioned study also confirm our results. However, the results of the research conducted by Mann et al showed no correlation between postpartum depression and spirituality in Latin-speaking women, which contradicts those of the present research, while spirituality reduced postpartum depression in English-speaking women. This research highlights the importance of social support and the quality of communication with pregnant women after accouchement (22). Based on the results of the present study, out of 152 studied women, 30 patients (19.8\%) obtained depression scores of 12 and higher, indicating that this malady has a high prevalence in the studied community. Based on the results of the above-mentioned study, the social and spiritual support of midwives reduces the stress in women with pre-eclampsia. Another study showed a positive and significant correlation between spiritual health and the quality of life in patients with thalassemia (23). In young people with thalassemia, increasing spiritual health is correlated with an increased quality of life and enhanced mental health. Accordingly, paying attention to the spiritual, physical, psychological, and social dimensions of patients is recommended to implement the holistic nursing care (24). A study conducted by Ngai et al on the physical and mental health demonstrated that spiritual and religious health and activity increase the odds of longer life and are considered as a health factor (25). It seems that organized religious involvement reduces postpartum depression symptoms (26).

In another study, EyvanBaga et al also reported that spiritual health can affect the incidence of mental disorders such as depression and anxiety. Based on the results of the study, paying attention to the spiritual health of the students as one of the factors affecting their health seems to be necessary (5). Previous evidence indicated that mental health is inversely associated with mood disorders and can be a preventive factor for these diseases. The results of the present research, in line with related studies, showed that spiritual health is associated with postpartum depression and can be considered as a factor for reducing and preventing postpartum depression. Hence, the incidence of depression and its costs and complications can be reduced by improving the spiritual health of women. The use of religious/spiritual resources in the coping process in the early stages of breast cancer plays a major role in the adaptation process in patients with breast cancer (4). The results of the studies by EyvanBaga et al (5) and ThunéBoyle et al (4) confirmed our results and revealed that the findings of most studies are in line with those of our study.

\section{Conclusion}

Based on the results of the present study, improving spiritual health as a complementary therapy, along with drug consumption can be used as a solution to prevent and reduce postpartum depression. Accordingly, it is suggested to introduce and recognize spiritual health as an important part of human being health. Eventually, other factors related to postpartum depression need further evaluation.

Conflict of Interests

None.

Acknowledgments

Hereby, we thank the Deputy of Research and Technology in Shahrekord University of Medical Sciences to support financially the project. We also would like appreciate all of individuals collaborated in the complement and performance of this project.

Ethical Considerations

This project was approved by the Ethics Committee of Shahrekord University of Medical Sciences under the code of IR.SKUMS. REC.1395.170. In addition, this thesis was approved by the code number of 1286 in Shahrekord University of Medical Sciences.

Authors Contribution

MN prepared the primary draft. LJ and SKS designed the study. MS completed statistical analyses. MB participated in data collection. AHD reviewed the final manuscript.

\section{Funding/Support}

Deputy of Research and Technology of Shahrekord University of Medical Sciences supported financially the project.

\section{References}

1. Nardi B, Laurenzi S, Di Nicolo M, Bellantuono C. Is the cognitive-behavioral therapy an effective intervention to prevent the postnatal depression? a critical review. Int J Psychiatry Med. 2012;43(3):211-25. doi: 10.2190/PM.43.3.b.

2. Habibzadeh A, Habibzadeh Z. Evaluation of effective factors and its prevalence on postpartum depression among women in the city of Qom, Iran. Int J Womens Health Reprod Sci. 2016;4(1):23-8. doi: 10.15296/ijwhr.2016.06.

3. Rahmani F, Seyedfatemi N, Asadollahi M, Seyedrasooli A. Predisposing factors of postpartum depression. Iran Journal of Nursing. 2011;24(72):78-87. [Persian].

4. Thuné-Boyle IC, Stygall J, Keshtgar MR, Davidson TI, Newman SP. Religious/spiritual coping resources and their relationship with adjustment in patients newly diagnosed with breast cancer in the UK. Psychooncology. 2013;22(3):646-58. doi: 10.1002/pon.3048.

5. EyvanBaga R, Nasiri K, Kamran A, Shamkhali R. The relationship between depression, anxiety and spiritual health among students of Khalkhal Faculty of Medicine Sciences. Community Health Journal. 2015;9(3):47-55. [Persian].

6. Striegel-Moore RH, Rosselli F, Perrin N, DeBar L, Wilson GT, May A, et al. Gender difference in the prevalence of eating disorder symptoms. Int J Eat Disord. 2009;42(5):471-4. doi: 10.1002/eat.20625.

7. Yim IS, Tanner Stapleton LR, Guardino CM, Hahn-Holbrook J, Dunkel Schetter C. Biological and psychosocial predictors of postpartum depression: systematic review and call for integration. Annu Rev Clin Psychol. 2015;11:99-137. doi: 10.1146/annurev-clinpsy-101414-020426. 
8. Jones HW, Venis JA. Identification and classification of postpartum psychiatric disorders. J Psychosoc Nurs Ment Health Serv. 2001;39(12):23-30.

9. Navarro P, García-Esteve L, Ascaso C, Aguado J, Gelabert E, Martín-Santos R. Non-psychotic psychiatric disorders after childbirth: prevalence and comorbidity in a community sample. J Affect Disord. 2008;109(1-2):171-6. doi: 10.1016/j. jad.2007.10.008.

10. Global, regional, and national burden of neurological disorders during 1990-2015: a systematic analysis for the Global Burden of Disease Study 2015. Lancet Neurol. 2017;16(11):877-97. doi: 10.1016/s1474-4422(17)30299-5.

11. Gentile S. Untreated depression during pregnancy: short- and long-term effects in offspring. A systematic review. Neuroscience. 2017;342:154-66. doi: 10.1016/j. neuroscience.2015.09.001.

12. Mohammad KI, Gamble J, Creedy DK. Prevalence and factors associated with the development of antenatal and postnatal depression among Jordanian women. Midwifery. 2011;27(6):e238-45. doi: 10.1016/j.midw.2010.10.008.

13. Timmins F, Neill F, Murphy M, Begley T, Sheaf G. Spiritual care competence for contemporary nursing practice: a quantitative exploration of the guidance provided by fundamental nursing textbooks. Nurse Educ Pract. 2015;15(6):485-91. doi: 10.1016/j.nepr.2015.02.007.

14. Puchalski C, Ferrell B, Virani R, Otis-Green S, Baird P, Bull J, et al. Improving the quality of spiritual care as a dimension of palliative care: the report of the Consensus Conference. J Palliat Med. 2009;12(10):885-904. doi: 10.1089/jpm.2009.0142.

15. Faribors B, Fatemeh A, Hamidreza $H$. The relationship between nurses' spiritual intelligence and happiness in Iran. Procedia Soc Behav Sci. 2010;5:1556-61. doi: 10.1016/j. sbspro.2010.07.325.

16. Smith LE, Greenberg JS, Seltzer MM. Social support and well-being at mid-life among mothers of adolescents and adults with autism spectrum disorders. J Autism Dev Disord. 2012;42(9):1818-26. doi: 10.1007/s10803-011-1420-9.

17. Azizi $M$, Faghihzadeh $S$, Nematollahzadeh $M$, Lamyian $M$. The effect of counseling on anxiety after traumatic childbirth in nulliparous women; a single blind randomized clinical trial. Behbood. 2010;14(3):219-27. [Persian].
18. Kozinszky Z, Dudas RB. Validation studies of the Edinburgh Postnatal Depression Scale for the antenatal period. J Affect Disord. 2015;176:95-105. doi: 10.1016/j.jad.2015.01.044.

19. Moghimian M, Salmani F. The study of correlation between spiritual well-being and hope in cancer patients referring to Seyyedo Shohada training-therapy center of Isfahan university of medical sciences, 2010, Isfahan, Iran. Qom University of Medical Sciences Journal. 2012;6(3):40-5. [Persian].

20. Abbasi M, Azizi F, Shamsi Goushaki A. Defining concept and safety of spiritual health: An cognitive study. 2012; 6 (20):1144.

21. Pourmohamadreza Tajrishi M, Alipour M, Chapari-Ilkhchi S. The effectiveness of conceptual map training method on the achievement of social-economic skill course in male students with intellectual disability. Iranian Rehabilitation Journal. 2013;11(1):46-52. [Persian].

22. Ramezankhan A, Ghaedi M, Hatami H, Taghdisi MH, Golmirzai J, Behzad A. Association between spiritual health and quality of life in patients with type 2 diabetes in Bandar Abbas, Iran. Hormozgan Medical Journal. 2014;18(3):210-8. [Persian].

23. Mann JR, Mannan J, Quiñones LA, Palmer AA, Torres M. Religion, spirituality, social support, and perceived stress in pregnant and postpartum Hispanic women. J Obstet Gynecol Neonatal Nurs. 2010;39(6):645-57. doi: 10.1111/j.15526909.2010.01188.x

24. Malakouti J, Sehhati F, Mirghafourvand M, Nahangi R. Relationship between health promoting lifestyle and perceived stress in pregnant women with preeclampsia. J Caring Sci. 2015;4(2):155-63. doi: 10.15171/jcs.2015.016.

25. Zeighami Mohammadi S, Tajvidi M, Ghazizadeh S. The relationship between spiritual well-being with quality of life and mental health of young adults with beta-thalassemia major. The Scientific Journal of Iranian Blood Transfusion Organization. 2014;11(2):147-54. [Persian].

26. Ngai FW, Wai-Chi Chan S, Ip WY. Predictors and correlates of maternal role competence and satisfaction. Nurs Res. 2010;59(3):185-93. doi: 10.1097/NNR.0b013e3181 dbb9ee.

27. Mann JR, McKeown RE, Bacon J, Vesselinov R, Bush F. Do antenatal religious and spiritual factors impact the risk of postpartum depressive symptoms? J Womens Health (Larchmt). 2008;17(5):745-55. doi: 10.1089/jwh.2007.0627. 